\title{
Heap profiling for space efficiency
}

\author{
Colin Runciman and Niklas Röjemo \\ Department of Computer Science, University of York, \\ Heslington, York, YO1 5DD, UK \\ (e-mail: \{colin, rojemo\}@cs.york.ac.uk)
}

\section{Introduction}

Excessive requirements for memory space have in the past hindered or even prevented otherwise attractive applications of functional programming. Although this could be blamed in part on space-hungry implementation methods, in most cases it would have been possible to cut memory requirements very significantly by making a few changes to the source program. But there were no tools to help programmers make appropriate changes. Usage of heap memory was reported only as a total volume of allocations; there was no way to investigate how different parts of a program made demands on heap memory - something which may be far from apparent in the source of a sizable program making use of lazy evaluation and higher-order functions. Finding the appropriate place to make a space-saving change could be very difficult.

In the last few years, there has been a renewed effort to provide appropriate profiling tools for functional programmers. Because the first aspect of performance that many software developers are concerned with is speed, many profiling tools are designed mainly to account for the use of execution time by different program components. But for current functional programming systems, memory-use is often more critical than processor-use. Memory-saving improvements typically save time too, whereas the reverse is less often the case. At any rate, our concern here is with profiling the use of heap memory.

A brief review of the development of memory-profiling systems for functional languages will be given towards the end of these notes. Apart from that, we concentrate throughout on explaining the concepts and use of the latest version of our own heap-profiling tools, as implemented in the nhc Haskell compiler. Perhaps this seems a little narrow-minded! By way of explanation: first, so far as we know the nhc profiler is the most advanced memory-profiling system currently available for a lazy functional language such as Haskell ${ }^{1}$; secondly, it suits our aim to give a practical tutorial with a series of worked examples and exercises. (We hope that all readers, like the participants at the Summer School, will have access to a computer system with nhc installed. Everything necessary can be obtained by FTP from ftp.cs.york.ac.uk under the directory nhc.)

\footnotetext{
1 Pace Glasgow! Their ghc compiler can profile both space and time, with cost centres to help localise faults, but has little support for classifying heap contents according to the dynamic characteristics of memory cells.
} 


\section{How to obtain nhc heap profiles}

To profile the heap usage of a Haskell program prog.hs using the nhc compiler, there are three steps.

1. Compile the program. Compilers that support heap-profiling typically require additional compile-time flags to request an executable with the potential to collect heap-profiling data at run-time. Making profiling an optional extra is usually appropriate because it does slow down the program. However, the version of nhc to be used in conjunction with these notes compiles all programs for heap profiling by default.

2. Run the program. Heap-profiling data of various kinds can be obtained by selecting an appropriate combination of run-time flags. At regular intervals, a census of the heap is taken, and profile data is written to a file prog.hp. By default, a census is taken every time a multiple of the heap size has been allocated. To request a different interval we use either -isizeb where size is the number of bytes allocated between censuses, or -itimes where time is the number of seconds between censuses.

3. Post-process the profiling data. The file prog.hp now contains the information we want, but not in a form that is easy to understand. The program hp2graph transforms .hp files into readable graphical charts (in PostScript by default). A command such as hp2graph prog.hp actually creates two new files, prog.ps and prog.aux. The former is a single-page PostScript file in which a graph of the live heap over time is automatically scaled to fill the page. The .aux file is useful if we wish to produce more than one graph on the same scale, for comparison purposes: hp2graph -pold.aux new.hp.

Steps 2 and 3 may be repeated several times, to obtain a variety of profiles. This may lead to a revision of the program, and restarting from step 1.

\subsection{An example: profiling the xref program}

Take as an example the xref program shown in Figure 1. The program reads text from the standard input, and writes on the standard output an ordered index to aid cross-reference; it lists all words in the input, and for each word the numbers of lines on which it occurs. The program is compiled by the command:

nhc -o xref xref.hs

To obtain profiles of the program running, we need to some test input. We shall use a 215-line file fplang 3 containing the first three messages sent to the 'FPlang' mailing-list (the start of discussions that led to the design of Haskell). 


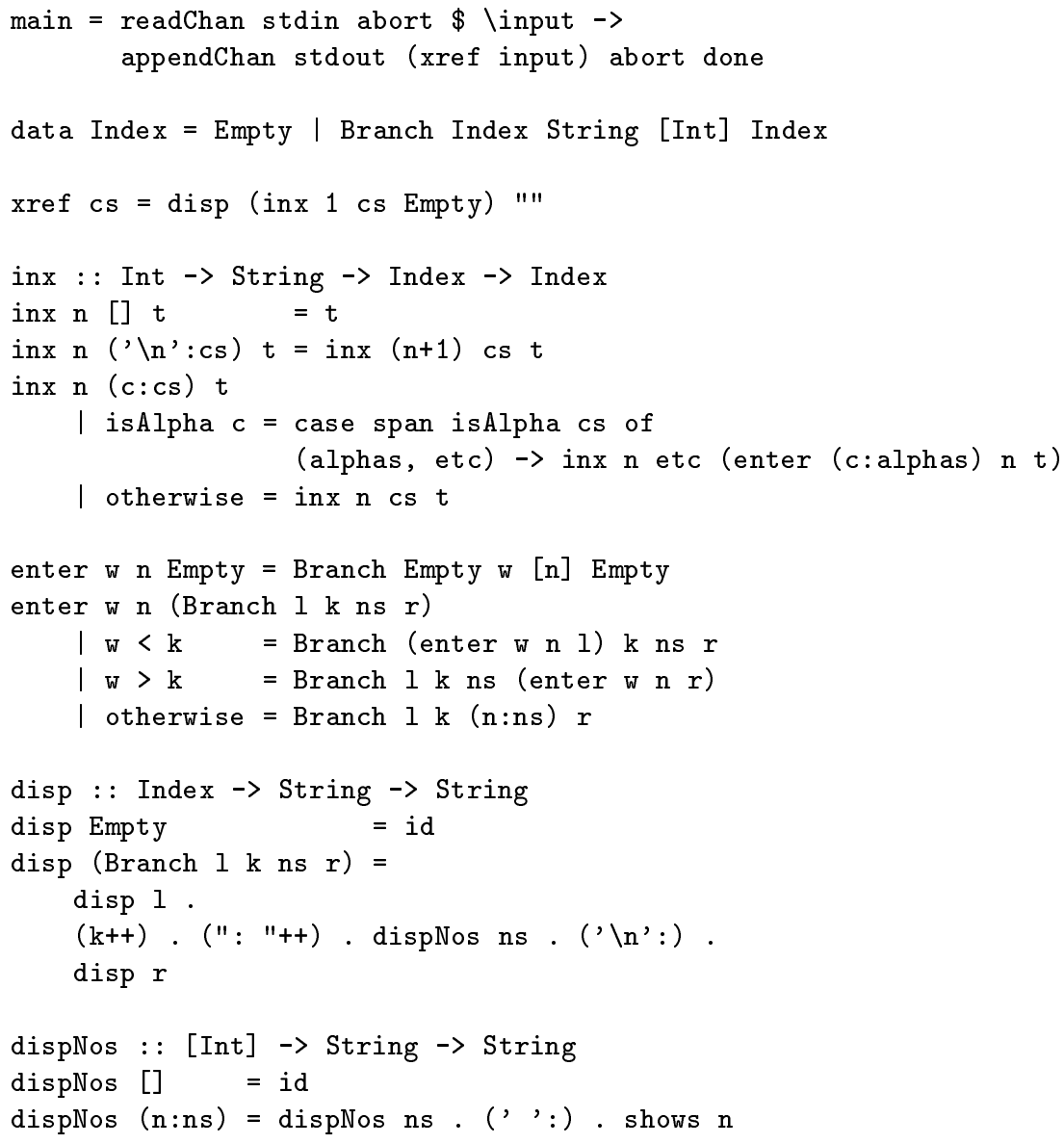

Fig. 1. The xref program.

\section{Producer profile}

Various run-time options request different forms of heap-profile. One of the most straightforward is a producer profile: it characterises cells in the heap by identifying which function produced them - ie. directly caused them to be allocated. A producer profile is requested by the $-\mathrm{p}$ option:

xref $-\mathrm{p}<$ input

We can now apply hp2graph to create the PostScript file xref.ps (and scaling information in xref .aux).

hp2graph xref.hp 


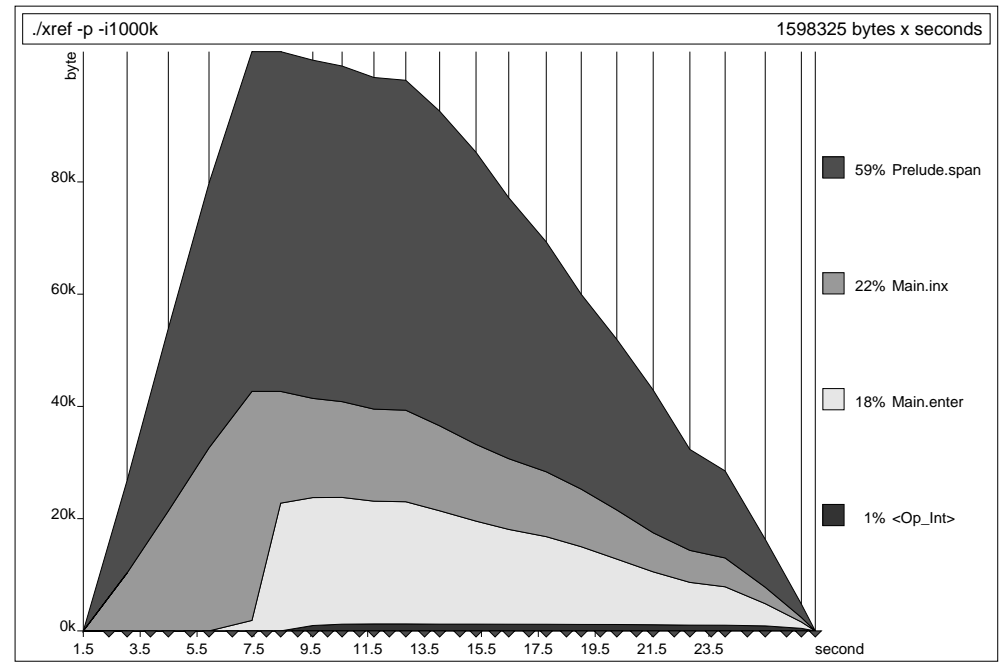

Fig. 2. A producer profile for xref.

The result of interpreting the PostScript in xref .ps is shown in Figure 2. Ignore the banding for the moment. Concentrate instead on the overall shape of the graph. The graph illustrates how the amount of live heap memory (vertical axis) varies over time (horizontal axis). In this example the live heap grows rapidly to over $100 \mathrm{~kb}$ before it starts to decrease. Although the collection of profiling data increases a program's memory requirements, this is not reflected in a heap profile - so the profile provides an accurate indication of how much heap memory is needed by the unprofiled program. The total execution time was slightly more than 25 seconds. Neither garbage-collection time nor heap-profiling overheads are included in this figure, so the wall-clock time for the profiling run may be much longer than the time shown in the heap profile. Also, execution times shown in heap profiles cannot be taken as accurate for unprofiled programs - though when comparing two programs, the ratio of their execution times is reasonably accurate.

The thin vertical lines mark the times when a census of the live heap was taken. Linear interpolation is used between censuses, which can be misleading for some programs. In doubtful cases the program can always be run again with more frequent censuses; there cannot be a hidden spike of more than $20 \mathrm{~kb}$ between censuses if the interval is $-i 20 \mathrm{~kb}$, for example. The triangles below the time axis mark garbage collections: every heap census forces a garbage collection, but there may also be collections at other times.

The title line contains the command line used when running the program (unfortunately without any redirections), and the total area of the graph. The latter can be viewed as a measure of the overall cost of the program in byte $\times$ seconds. 


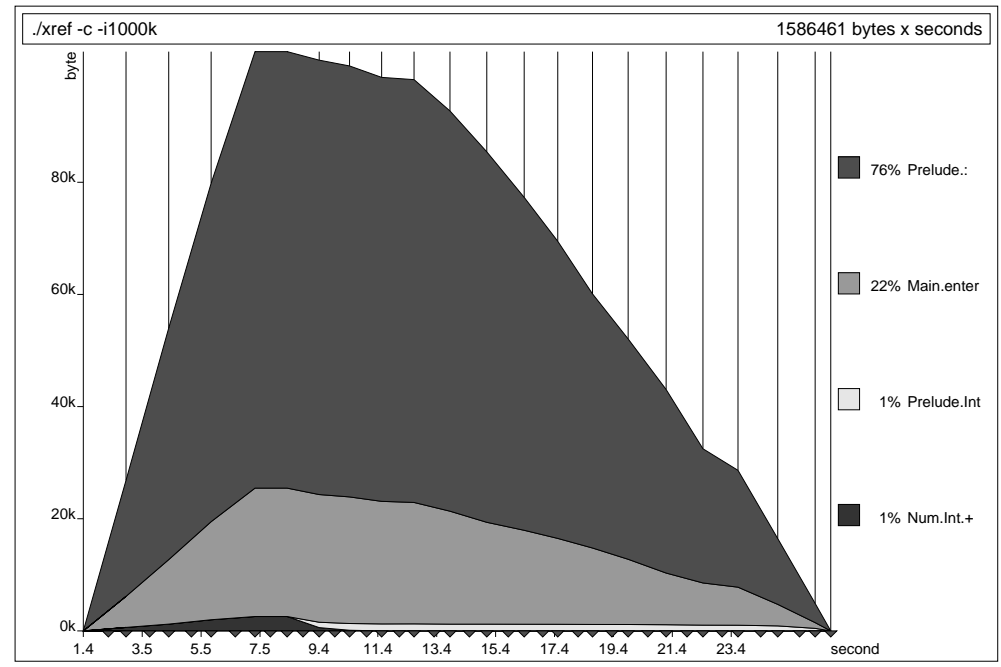

Fig. 3. A constructor/closure profile for xref.

To the right is a list of keys, describing what each shaded band in the graph stands for. The example in Figure 2 is a producer profile so there is a shaded band for each different program component that allocates memory. In our example, the function span in the module Prelude was the main allocator of heap memory. The percentages indicate how much of the overall cost is attributed to each key. Percentages are rounded to the nearest whole number, so they do not always sum to 100 . The key <0p_Int $>$ marks memory allocated by primitive functions working on machine words. If there are more distinct keys in a heap profile than can fit in the graph, then the keys with smallest area are collapsed into an OTHER key.

It is possible to obtain a coarser form of producer profile, in which producers are whole modules by using the command xref $-\mathrm{m}<$ input. This can be very useful for large programs, but would in our example only join Main.inx and Main.enter into one key, Main.

\section{Constructor/closure profile}

A useful complement to a producer profile (and in older heap profiling systems the only other possibility) is a constructor/closure profile. After the run:

xref $-c<$ input

hp2graph produces the chart shown in Figure 3. The overall shape and cost are almost the same as before - they differ very slightly because of small variations in timing. Now each key represents a different kind of cell: a constructor profile 


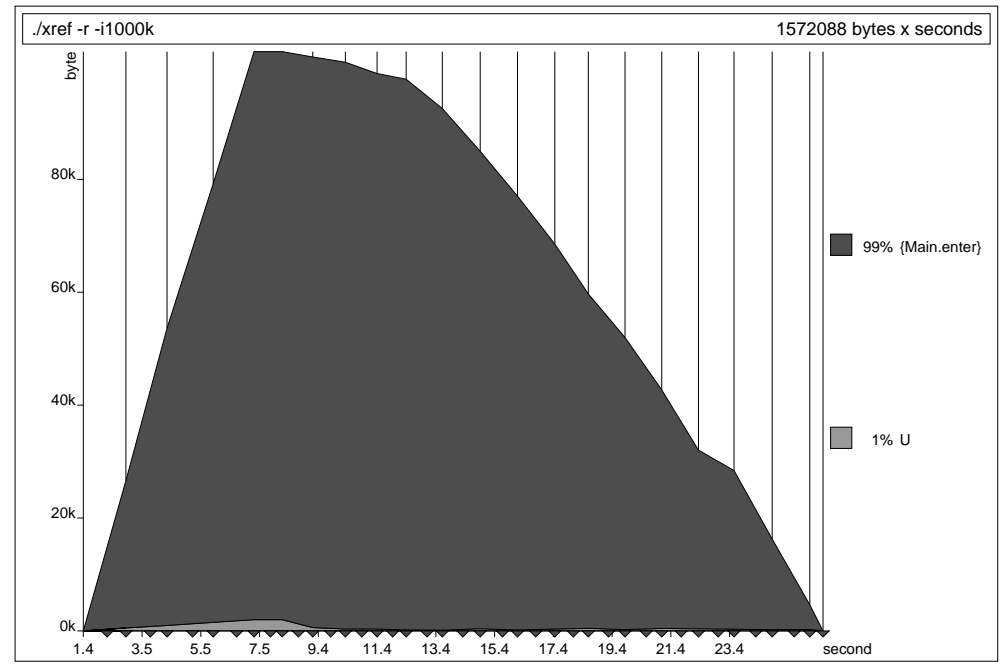

Fig. 4. A retainer profile for xref.

characterises a heap cell by asking 'What is it?'. In our example $76 \%$ of the overall cost is accounted for by 'cons' cells (Prelude.:). Perhaps surprisingly, there are no cells representing the constructors of the Index tree: we shall return to this observation in $\S 4.5$.

Names of functions other than constructors can appear among the keys, indicating that memory is used for unevaluated closures. For example, Figure 3 includes a band for closures of the function Main. enter. Closures of functions that are defined as part of a type-class instance are indicated by a three-part name of the form class. instance. method. For example, $1 \%$ is allocated to closures of the + method for the Int instance of the class Num.

\section{Retainer profile}

A retainer profile characterises each heap cell according to the program components that have immediate access to it, perhaps as part of a larger data structure. After the command:

xref $-r<$ input

hp2graph generates the retainer profile shown in Figure 4 showing that closures of enter retain almost the entire heap. Notice that the keys are now sets. A heap cell can be retained by closures of more than one function due to sharing. By default all sets with more than one member are collapsed into one set. By abuse of terminology this is called the universal set $\mathrm{U}$. To split the U-band, give an integer after the $r$ flag: for example, xref $-r 3<$ input distinguishes 
retainer sets up to size 3 . Sets larger than the given limit are still combined into a universal set.

Program components that can occur as retainers are of two kinds. First there are functions whose applications, until fully-evaluated, may retain heap cells in their arguments. Secondly, there are so-called constant applicative forms (or CAFs): named data structures defined at the top-level of the program.

\section{Biographical profile}

The final kind of heap profile provided by nhc is a biographical profile, which characterises heap cells according to their past, present and future usefulness in the computation. After running the xref program by:

xref $-\mathrm{b}<$ input

hp2graph gives the profile shown in Figure 5.

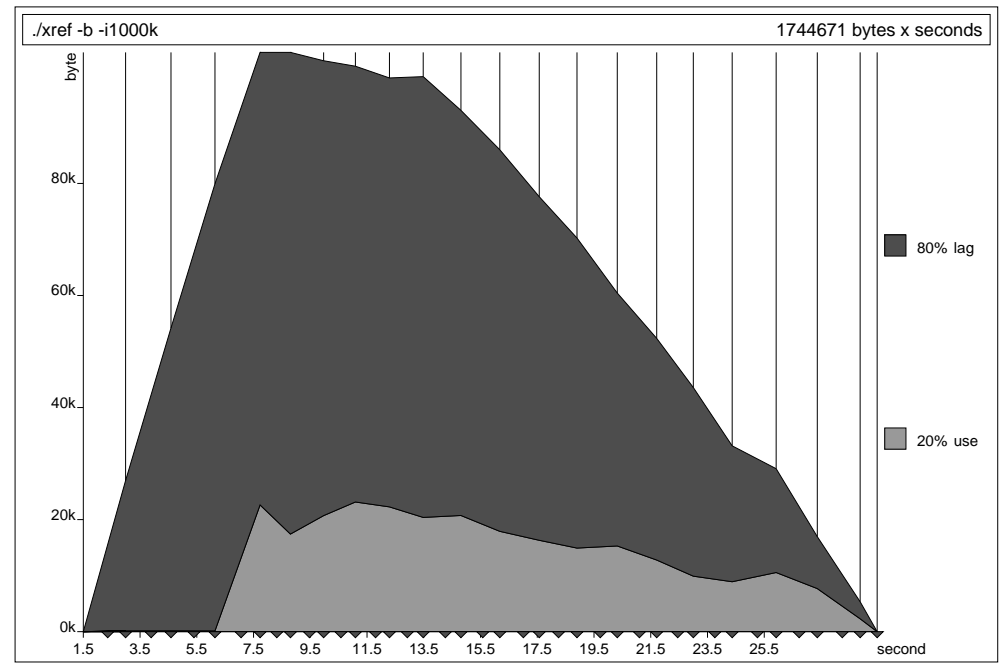

Fig. 5. A biographical profile for xref.

In general, a biographical profile splits the heap into four bands (although only two appear in our example). Cells that are never used are void. If a cell is used then it passes through three phases: it is lag between creation and first usage, use between the first and the last usage, and finally drag between the last use and its destruction.

\begin{tabular}{|c|c|c|}
\hline Cell used in the past? & yes & no \\
\hline Cell used in the future? & \begin{tabular}{l|l|} 
yes & no \\
\end{tabular} & \begin{tabular}{|l|l|} 
yes & no \\
\end{tabular} \\
\hline 8011 & & $l_{0}$ \\
\hline
\end{tabular}


A cell is considered used when it is scrutinised in a case, or taken part in a primitive operation, or updated with its result (only possible for function closures).

One big difference between the biographical profile and the others is the lack of references to the source code. Other profiles have keys that identify parts of the source program, but the keys in a biographical profile are just cell phases. However, the unique advantage of a biographical profile is that it may directly point to an apparent waste of heap memory in the $d r a g$ and void bands, and perhaps in the lag band too - the xref program, for example, seems to create a lot of heap cells long before they are needed (see $§ 4.5)$.

\subsection{Choosing the right profile}

Often one resorts to a heap-profiler with a question already in mind, and that determines the kind of profile needed. For example, the question 'Which is taking up more heap-space, the lists or the trees?' would be answered by a constructor/closure profile.

But what about the outset of a general examination of space-efficiency in a program? Which kind of profile should be looked at first?

One tactic is to obtain all four, and then proceed by investigating the most puzzling of the large bands or spikes. However, we have found that a biographical profile often gives the most useful overall picture of heap-use, without identifying specific program components. A very large percentage of drag, void or lag is often the symptom of a space-fault, and the offending category of cells should be studied further by one of the profiling methods that identifies program components. Such investigations of parts of the heap only are made by specifying restrictions among the run-time profiling options. Examples will be seen in $\S 4$, and summary tables of available options are given in an appendix.

There is an important caveat to the tactic of beginning with a biographical profile: even if it shows that all cells in the live heap are in their use phase, this is not a proof of an efficient program. The program might just use a poor algorithm which re-evaluates everything several times!

\section{Causes of space-inefficiency}

Before we look any further into the space-efficiency of particular programs, we next give a brief statement in general terms of the typical sources of spaceinefficiency in functional programs. We can divide these into three categories.

1. Degree of evaluation. An often-made criticism of lazy evaluation is that without very careful programming it can lead to a large number of unnecessarily suspended computations taking up a lot of heap space. The criticism is valid, and one use of a heap-profiler is to check whether such a fault is present so that, if so, it can be remedied. However, it is equally the case that eager evaluation can lead to unnecessary computations whose results fill the heap. Even in a 'lazy' language one can define functions in a way that makes them unnecessarily strict. 
2. Degree of sharing. Sharing is another two-edged sword. One might think that shared reference to a single structure would always give better spaceefficiency than reference to multiple versions of the same value. But the 'same value' when shared must also be evaluated to the same extent: once evaluated because of the demands of one reference, a large shared structure must remain in heap pending its access by other references. By contrast, the pattern of demand in the evaluation of an unshared structure may allow it to be traversed in constant space (unevaluated 'in front' of current references, and garbage-collected 'behind' them).

3. Representation and algorithmic choices. These choices are often closely connected with the two previous issues, but can also stand alone as sources of space-inefficiency. Even in the stylised world of sum-of-products data types, there are more-compact and less-compact alternatives. The effect of an algorithm choice on space-efficiency often has to do with the order in which parts of the computation are done: advancing or delaying the use of a component function may be one way to avoid long-lived large structures, for example.

There is no fixed association between each source of space-inefficiency and a single kind of profile by which it is detected. A degree-of-evaluation problem, for example, might be apparent either in a high proportion of closures in a constructor/closure profile or in a large lag component in a biographical profile. However, only retainer profiling yields direct information about sharing.

It is beyond the scope of these notes to discuss implementation aspects of space-inefficiency in any detail, though the space-efficiency of some functional programs is certainly compiler-dependent. Beware!

\section{A series of examples}

In this the major section of the notes we shall examine various aspects of heap profiling and space efficiency as they arise in a series of example programs. Like xref, all the programs are small utilities that take text files as input; some are loosely based on UNIX tools. Profiles throughout are of runs with the 215-line file fplang3 as input.

\subsection{The cat program}

We begin with something very simple that already makes efficient use of heap space. The cat program of Figure 6 prints its input as its output. Figure 7 shows a heap profile of cat running with the 'FPlang' sample as input. We note with pleasure that cat runs using a small and almost constant amount of heap-space - about 200 bytes. What exactly do those 200 bytes contain? The constructor/closure profile of Figure 7 gives the answer: about half the space is occupied by cons $(:)$ cells forming the spine of the lazy list of characters representing the text. As the input is lazily demanded for consumption as output, each 


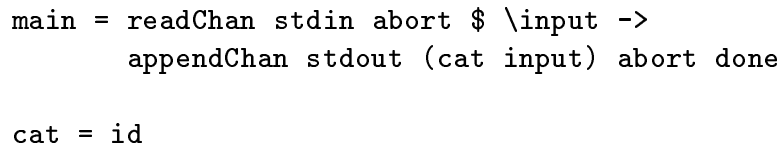

Fig. 6. The cat program.

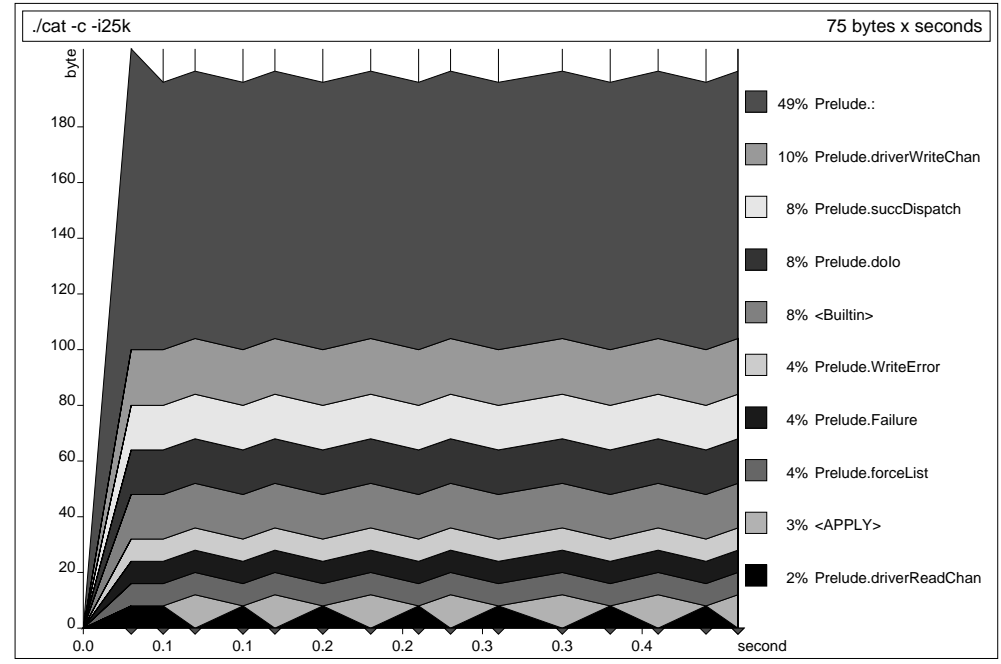

Fig. 7. A constructor/closure profile of cat.

cell is short-lived and only a tiny fraction of the spine for the whole text is present in the heap at any time. The rest of the live heap is accounted for by primitive auxiliaries such as Prelude.driverReadChan and Prelude.driverWriteChan that deal with input and output. Individual characters do not appear: there is exactly one copy of each distinct character constant, stored outside the heap.

Exercise The function lines : : String $\rightarrow$ [String] breaks text into a list of lines; the inverse function is unlines. So one should be able to replace id in the cat program by unlines. lines. Both functions are defined in the Haskell prelude, but try defining your own equivalents. Check that their composition can be used in cat without upsetting its space behaviour (apart from a modest constant overhead).

\subsection{The trail program}

Our next example can be viewed as a refinement of the cat program. Instead of the whole of the input, the output of trail is only the trailing $\mathrm{n}$ lines of input, 
where $\mathrm{n}$ is an argument to the program ${ }^{2}$.

One popular way to approach such a problem is to decompose it into a functional pipeline. The auxiliaries lines and unlines let us transfer between lists of lines and lists of characters. The asymmetric cons-list representation makes it awkward to extract $\mathrm{n}$ lines from the back of a list. But to extract $\mathrm{n}$ items from the front is straightforward using the auxiliary take; so let's reduce our problem to this case by applying reverse to both input and output. Hence we obtain the program in Figure 8.

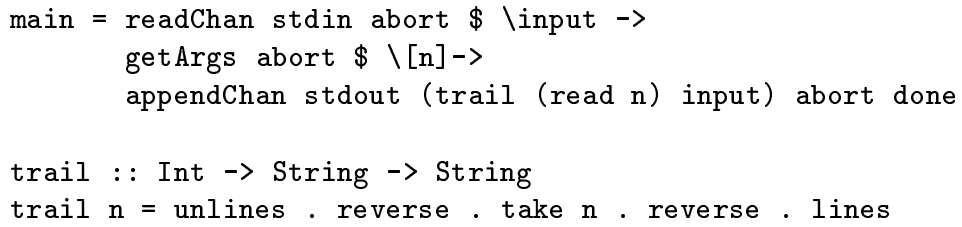

Fig. 8. A prototype trail program.

But is it space-efficient? Applying trail -b 50 with fplang3 as input gives the biographical profile in Figure 9. The live heap grows to about $100 \mathrm{~kb}$. The bulk of it is occupied by void cells, with a significant minority in the lag phase. Seeking an explanation in terms of program components, we apply retainer profiling to each of the void and lag components. The profiles show that void cells are retained by reverse (and subsequently take) on the input side (Figure 10); and similarly that the lag cells are retained by reverse (and subsequently unlines) on the output side.

The use of a recursively-strict function over a data structure in a compositional pipeline is a standard pitfall. It negates the space-efficiency of lazy evaluation by forcing an unbounded amount of data structure into live heap. The reverse function is a classic example. There are various techniques for avoiding this sort of problem. A change of representation is not an option for the trail program. But a revised algorithm can make simultaneous access to a whole structure and some part(s) of it, as yet unevaluated, in order to combine structure-creating computations with structure-discarding ones. In conventional terms, we maintain two pointers into the file. See Figure 11 for the new program. Figure 12 shows a profile with the same input and scale as before. It might seem that the program still has unduly large void and lag components, but this is not the case. The program maintains a bounded buffer containing the required number of lines of text. The buffer contents are void until the final section of the input is reached; this final section builds up as lag in the buffer until it is printed.

${ }^{2}$ So it is a version of the UNIX utility tail, but renamed to avoid a clash with Haskell's prelude function tail that extracts the tail of list. 


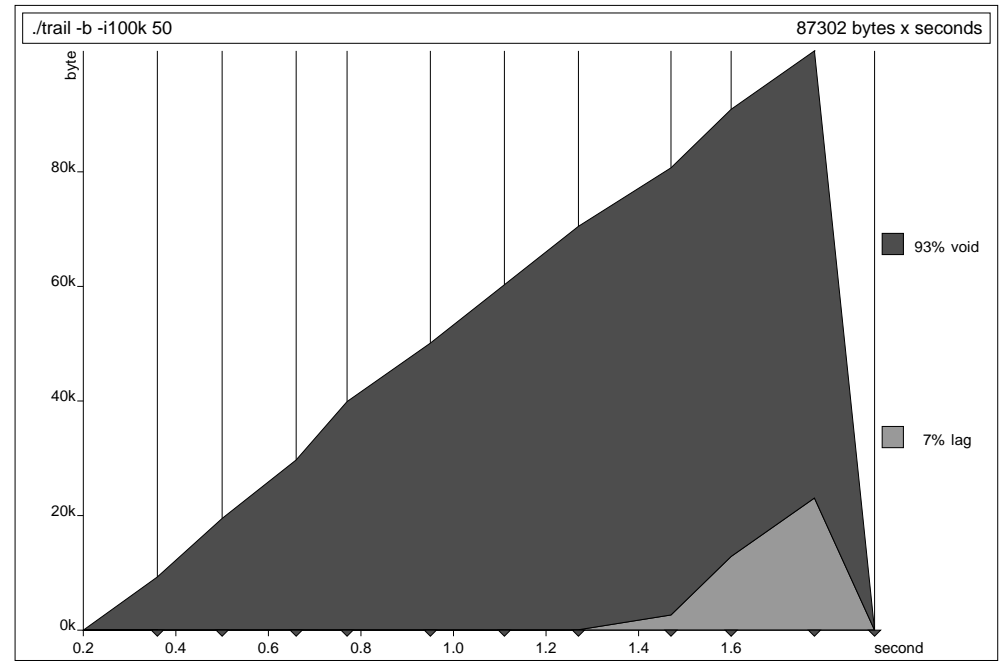

Fig. 9. Biographical profile of the trail prototype.

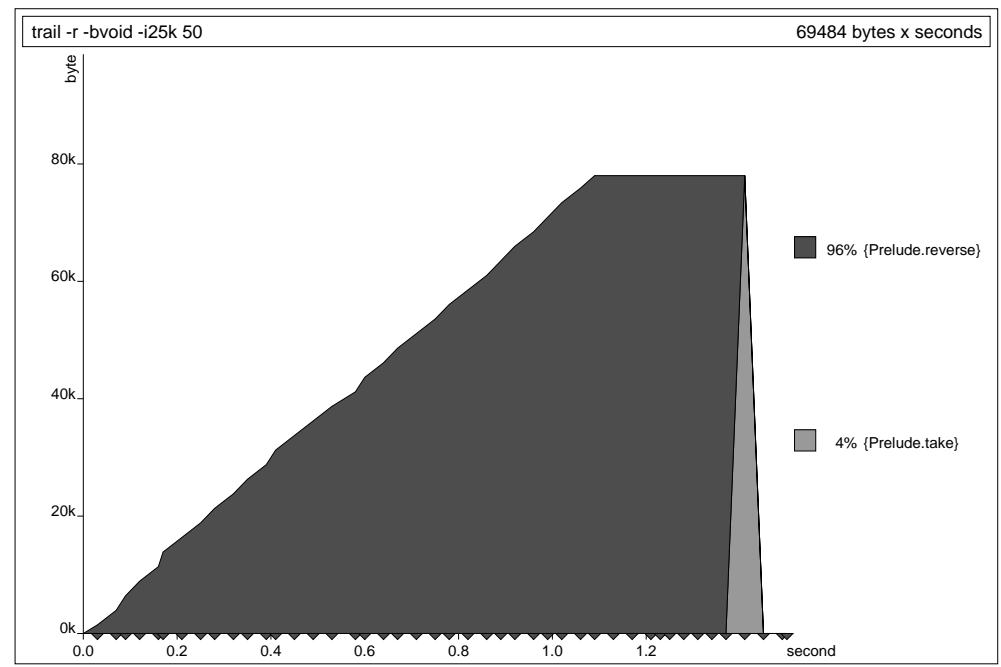

Fig. 10. A retainer profile for the heap void of the trail prototype. 


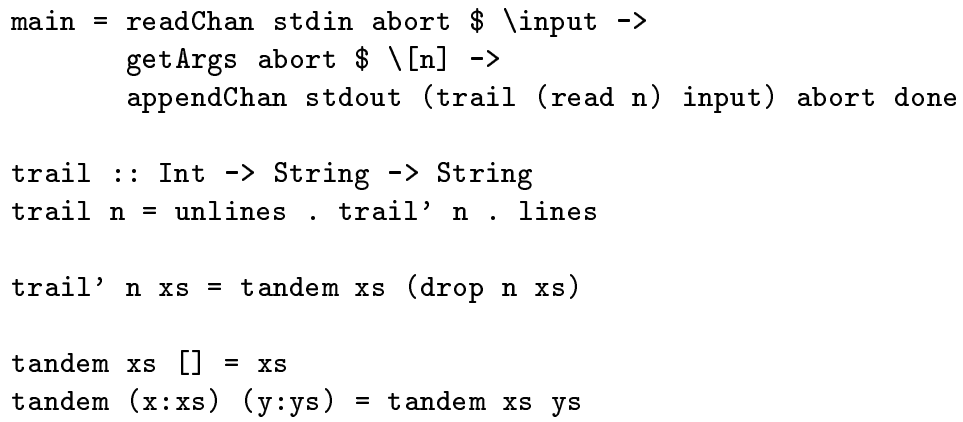

Fig. 11. An improved version of trail.

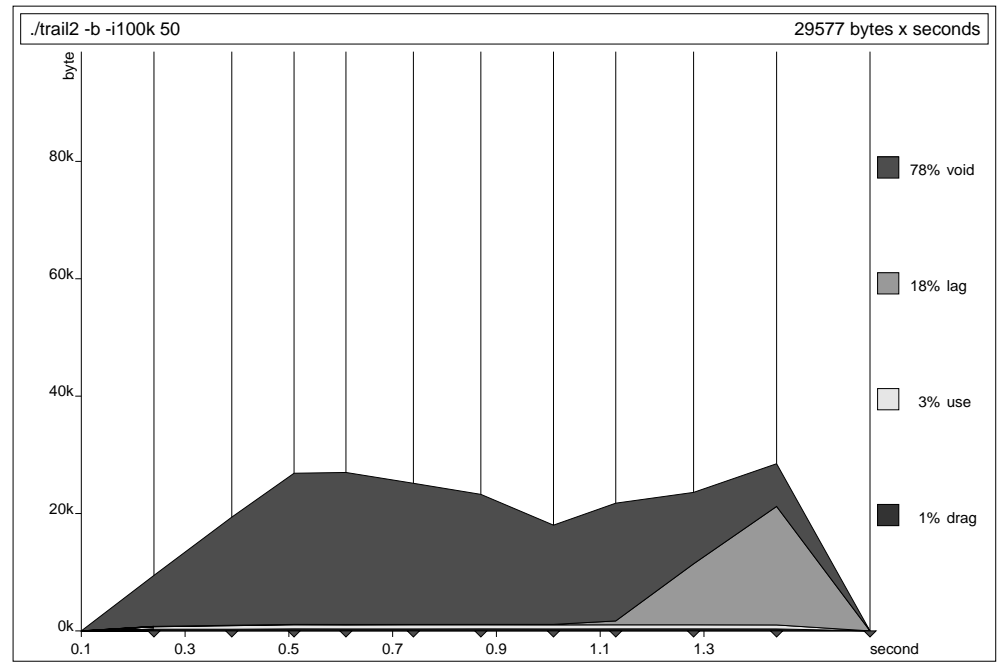

Fig. 12. An improved trail profile (cf. Figure 9).

One last puzzle: why does live-heap size dip in the middle of the computation? Because the author of the second contribution to 'FPlang' wrote in short lines!

\subsection{The maxw program.}

The maxw program lists the longest words it can find in its input, in alphabetical order.

Again we can tackle the problem as one of composing appropriate elements in a pipeline, keeping in mind that because of lazy evaluation the computations of pipeline elements do not necessarily occur in strict sequence. A prototype pro- 


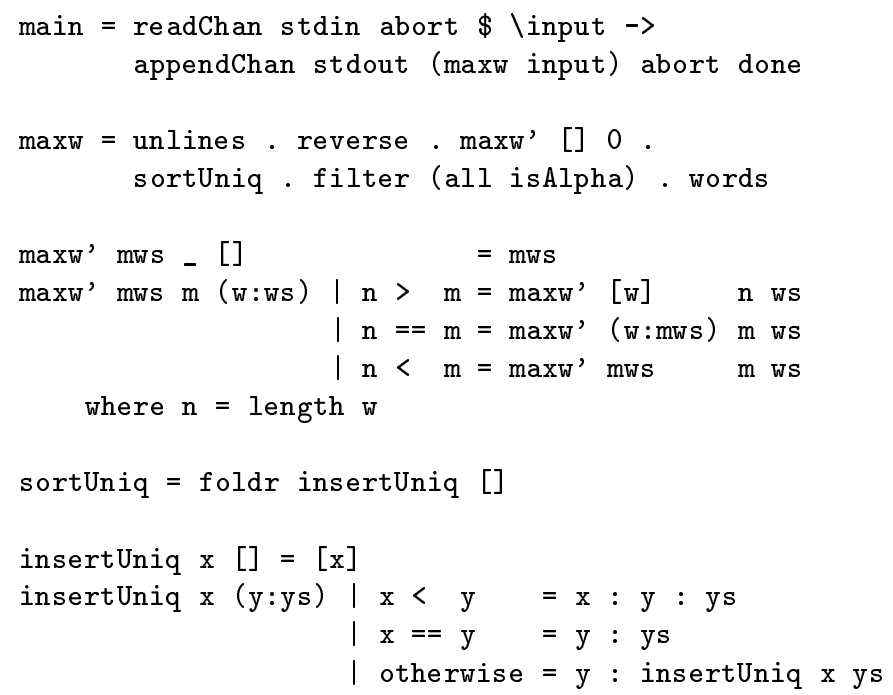

Fig. 13. The prototype maxw program.

gram is shown in Figure 13. From the input we extract words: using the standard words auxiliary we actually obtain more than that - it gives all maximal sequences of non-space characters - so the next element is a filter selecting the alphabetic strings only. A sortUniq at this stage also enables duplicates to be discovered and discarded. A list of the longest words is extracted and unlines prints them one-per-line.

Exercise The prototype maxw program is far from space-efficient: can you predict the shape of its heap profile? There is a remedy without any major rewriting of the program; only a couple of minor changes are needed. With the aid of the heap profiler, obtain and verify much-improved space-efficiency (and speed) in a revised version of the program. Is the revised program better for all inputs?

\subsection{The wc program}

The UNIX wc (for word-count) program does slightly more than its name suggests. It actually counts the numbers of lines, words and characters in its input, which may be specified as named files. Our version will do the same, but for the standard input only.

Figure 14 shows a naive prototype, in which the three required counts are computed by three separate expressions over the input. This is not a spaceefficient solution: because of the shared references to the list cs of input characters, as the first expression is evaluated the entire spine of cs is forced into heap memory. It cannot be discarded as the first computation advances through it, 


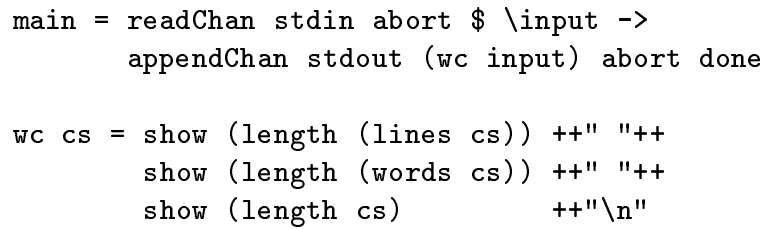

Fig. 14. A naive wc program.

because it will also be needed by the other two. A retainer profile for cons-cells (Figure 15) shows the effect. The auxiliary functions break and dropWhile are used in the Prelude definitions of lines and words. The two dark spikes occur because in a small number of censuses, words had just passed the remainder of the file contents to its auxiliary dropWhile: a profile with fine enough intervals would show many more such spikes. Note: since the retained spine is used again

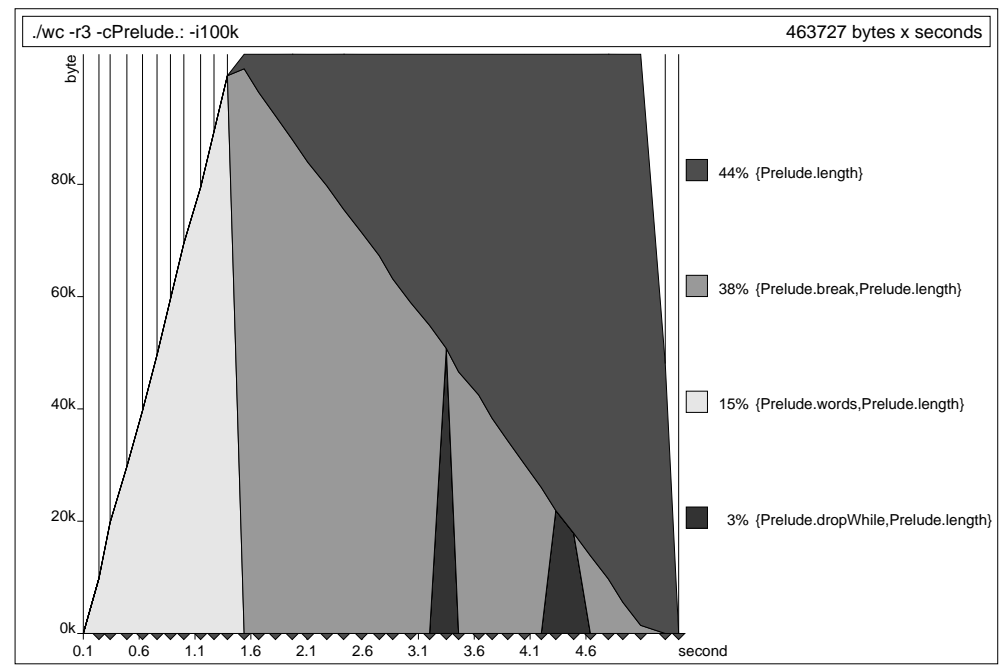

Fig. 15. The cons-cell retainers in naive wc.

later, a biographical profile would not in this case point to wasted memory.

Aiming for a program that runs in a small constant space for all inputs, we rewrite wc as in Figure 16. This program makes a single traversal of the input, checking for both word and line boundaries. The first argument of the tailrecursive auxiliary wc' is a triple accumulator for the line, word and character counts. 


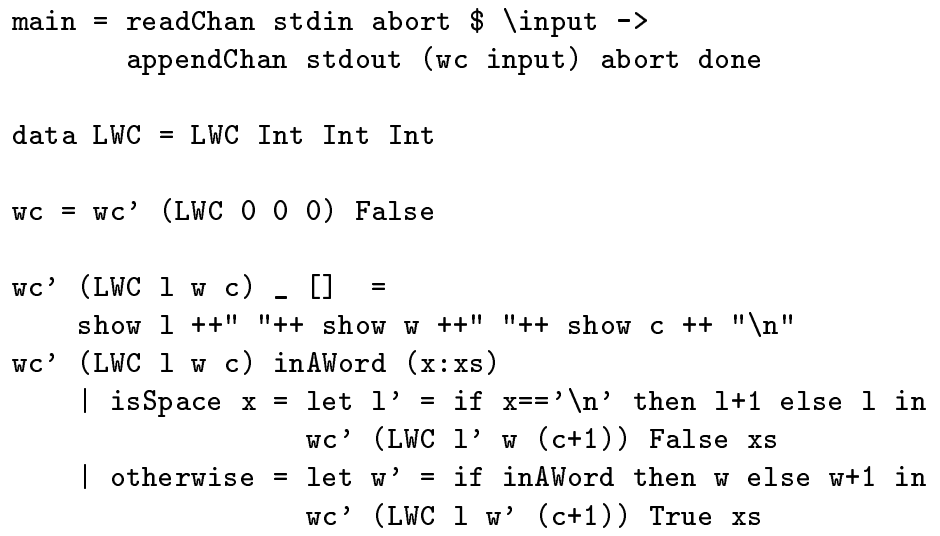

Fig. 16. A single-pass wc program.

Is this program space-efficient? No! A biographical profile shows a heap that grows to a peak size almost twice that of the 'naive' wc, only diminishing in the last $10 \%$ of execution time. To the nearest $1 \%$ the heap is $100 \%$ lag! This suggests an accumulation of unevaluated closures, confirmed by a constructor/closure profile for the heap lag (Figure 17). Unevaluated additions and other computations from the body of $\mathrm{wc}^{, 3}$ fill up the heap. No further profile is needed to determine where they accumulate; it can only be in the LWC counters.

This is the kind of space-fault that can discourage one from using a lazy functional language, even though the use of laziness can also be elegant and effective. Of course, it can save space to delay the evaluation of a recursivelystructured value; the structure may be larger than the closure that computes it. But it never saves space to delay the evaluation of a basic value such as an integer - though it may save time if the integer turns out not to be needed. In the wc program, all three integers of the LWC accumulator are needed, so they are better evaluated strictly. In Haskell, strict evaluation can be specified by adding !-annotations to the Int components in the definition of LWC.

data LWC = LWC ! Int ! Int ! Int

Now if we re-run the program and print a profile to the same scale, the live heap almost vanishes, barely exceeding 350 bytes.

\subsection{The xref program (resumed).}

Reviewing the heap profiles of the prototype xref program in $\S 2$, we make the following observations.

\footnotetext{
3 These 'other computations' are actually the conditional increments.
} 


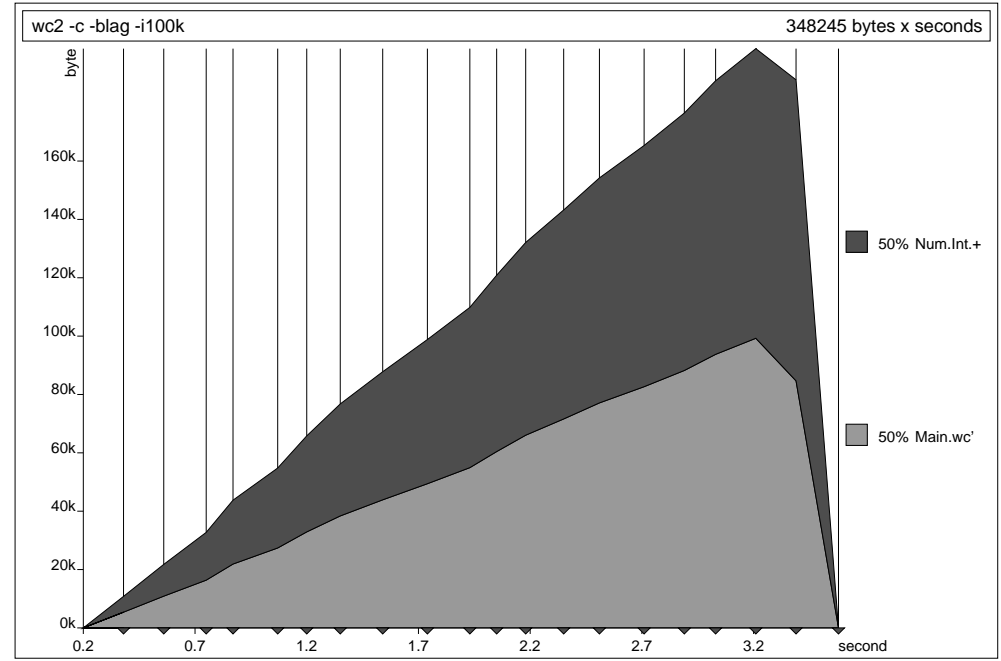

Fig. 17. Lazy accumulation; closures lagging in wc.

1. In the constructor/closure profile (Figure 3), as expected there are many cons-cells forming list spines. But there is no sign of constructors for the cells representing the constructors of the Index tree; and almost a fifth of the space is occupied by enter closures.

2. In the retainer profile (Figure 4) enter closures account for the retention of almost the entire heap.

3. In the biographical profile (Figure 5) most of the heap is lag.

It seems that we may be losing space-efficiency because computation is delayed. The function enter inserts occurrences of a word into the Index tree. But because evaluation is lazy, successive applications of enter simply extend a chain of closures. The Index tree is only built when the time comes to print it - it does not show up in the heap profile because each piece is discarded as soon as it has been printed.

Our preferred order for the main computational events is clear: as each word is reached in the input text it should be transferred to an entry in the index tree, so that only one copy of each word need be retained. Continuation-passing is a standard technique for expressing sequence in functional programs. To ensure that application of a function $f$ occurs after an enter computation is complete, we make $f$ an additional argument to enter, redefining it like this: 


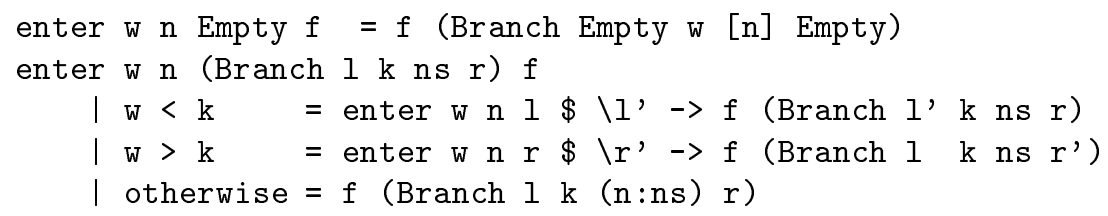

Correspondingly, the call to enter from inx becomes:

enter (c:alphas) $n$ t $\$$ inx $n$ etc

These changes ensure that enter records each word in the Index tree before inx reads the next. Figure 18 shows a constructor profile of the new program, on the same scale as Figure 3. Overall cost is reduced by about $25 \%$. The enter closures are gone, replaced by constructors of the index tree; and there is less list structure representing words of the text. A biographical profile would show

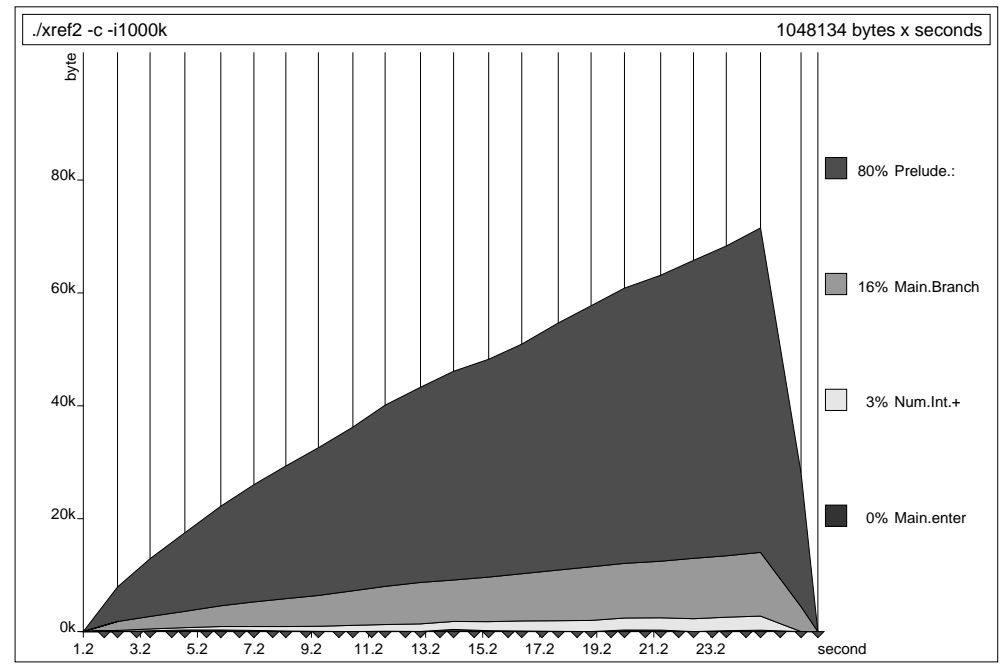

Fig. 18. A constructor/closure profile of improved xref.

that there still is quite a lot of lag: much of the Index tree, including all the recorded line numbers, will only be used in the final stage of printing.

Exercise As seen in the wc example, strictness annotations can also be used to bring computation forward. What is the effect of introducing strictness annotations in the definition of the Index type in the original xref program? Can this be the basis of a similar improvement in space efficiency? 


\subsection{The diff program.}

Our final example is diff, a file-comparison program. The problem of listing the differences between files is often tackled using simple rules of thumb - fast to compute but not guaranteed to find the least expression of differences. This version of diff gives as output a minimal sequence of editing steps that if applied to the first file would make it identical to the second.

The program in Figure 19 is based on one given by Allison [1] to compute distances between strings. Definitions of main and format have been omitted to fit the program on the page: main simply reads two files whose names are arguments to the program, and prints the result of applying diff to the file contents; format is of type [Edit]->String, and constructs the output representation of a sequence of edits.

The central idea is to compute a matrix of correction sequences by dynamic programming. For an application diff file1 file2, with $M$ lines in file1 and $\mathrm{N}$ in $\mathrm{file} 2$, the matrix has rows $0 \ldots \mathrm{M}$ and columns $0 \ldots \mathrm{N}$. The element at row $\mathrm{m}$ and column $\mathrm{n}$ is the minimal correction sequence between lines $1 . . \mathrm{m}$ of file1 and lines $1 . . \mathrm{n}$ of file2. In particular, the desired result is the sequence at $(\mathrm{M}, \mathrm{N})$. The elements of the matrix are computed recursively. As the base case, the correction sequence at $(0,0)$ is empty; otherwise at $(0, n)$ it adds at the start of file1 the first $n$ lines of file2, and at $(m, 0)$ it deletes the first $m$ lines of $f$ ile1. For positive $(m, n)$, if the files differ at those lines, the correction sequence is obtained by appropriately extending a shortest sequence from those at $(m-1, n),(m-1, n-1)$ and $(m, n-1)$; if the lines do not differ the correction sequence is just that at $(m-1, n-1)$.

Because a line-by-line simultaneous advance through each file corresponds to a diagonal path in the matrix, the matrix is most conveniently represented as a collection of diagonals. We define the principal diagonal prince, and two further lists of diagonals (uppers and lowers) each ordered by increasing distance from the principal.

Figure 20 shows the biographical heap profile of the program in Figure 19 applied to our usual 215-line trio of mail messages and a corrupted version of them with one change (in line 138). The space consumption is monstrous! A step-by-step investigation by successive heap profiles would be too extensive to include here. But in this case Allison has already made the critical observation in [1]: the matrix should only be computed by need. If the two files are in fact identical we need no more than the principal diagonal. More generally, we should be careful not to demand the evaluation of diagonals to any greater extent than is strictly necessary. For just this reason head and tail are used in the body of diagTails rather than deeper pattern-matching on the left-hand side. To improve space-efficiency we must do the same in diag, revising the final equation in its definition as follows:

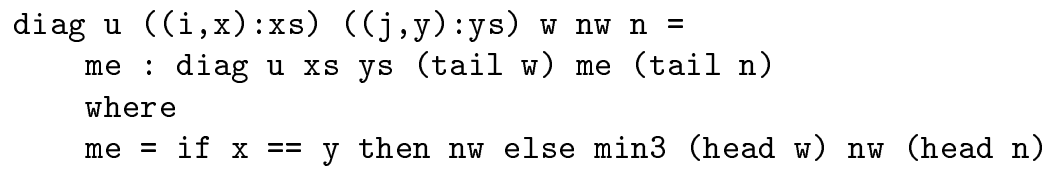




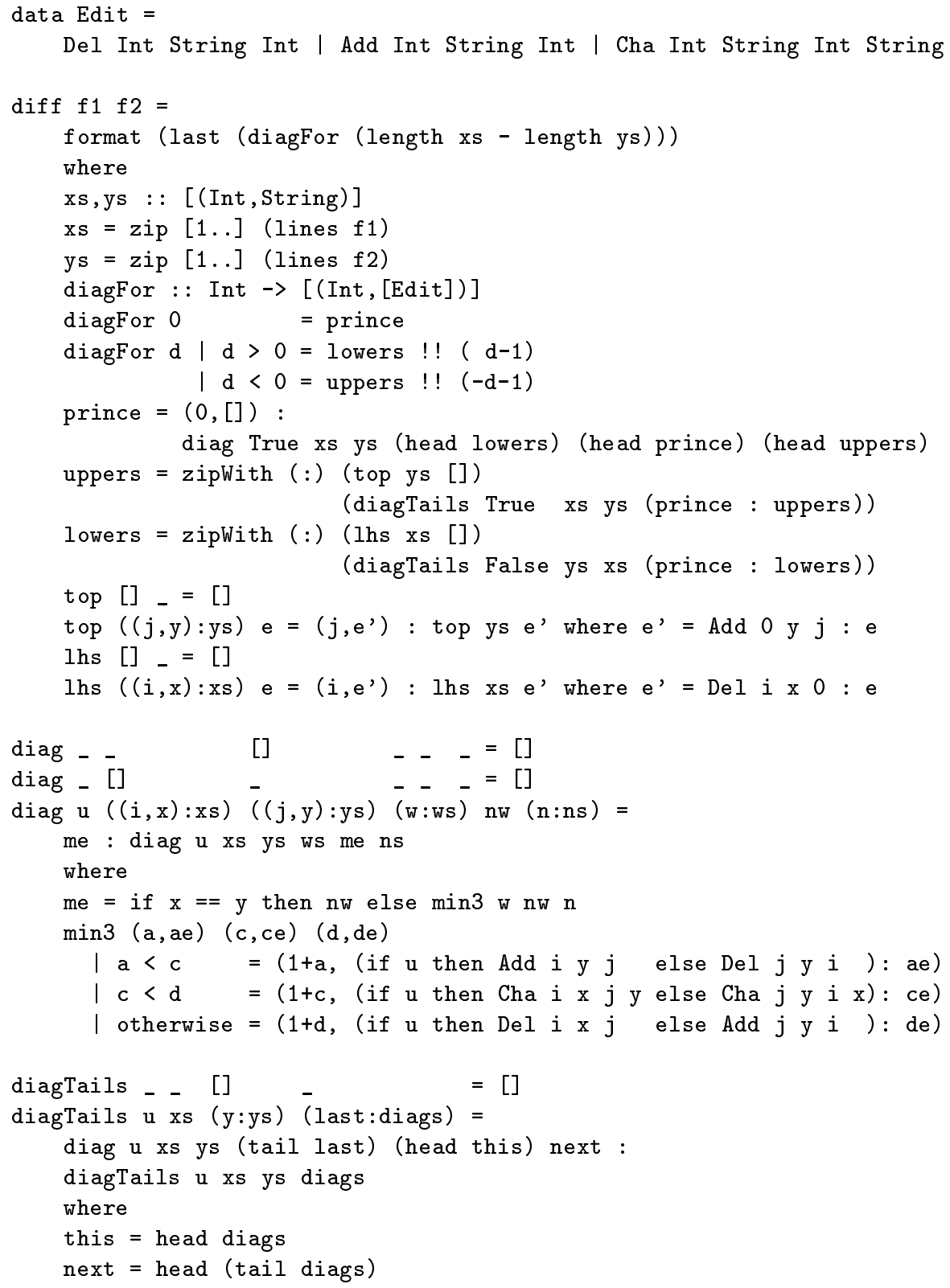

Fig. 19. A diff program. 


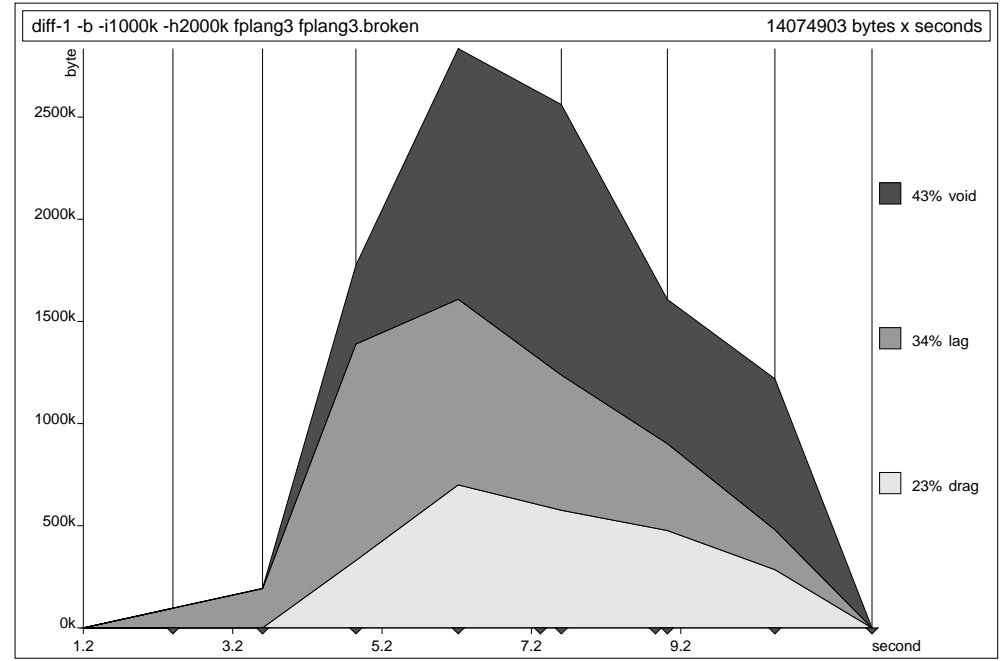

Fig. 20. A monster profile: diff at work.

Now if we repeat the previous diff application, we obtain the profile of Figure 21 (plotted to the same scale). Making the evaluation lazier has saved a lot of space.

Exercises Though only a fraction of the size of the more eager diff's live heap, the live heap even in the improved version remains large. In part this is an inevitable consequence of insisting on minimal edit sequences, but perhaps there is still scope for improvement.

1. Early in the computation the length of each file is computed, forcing the full contents of both files into heap memory, where they remain as they will be needed again. Investigate the effect on heap-use of re-reading the files instead.

2. Use heap-profiling with restrictions to characterise each of the void, drag and lag components of the heap. Can you further reduce the volume of cells in any of these phases?

\section{A brief history of heap-profiling}

Programming languages with heap-based implementations are hardly new. Yet, whereas many such implementations provide profilers for execution-time, we know of very little development and use of tools to examine the make-up of heap memory during a computation. We do not count profilers that measure 


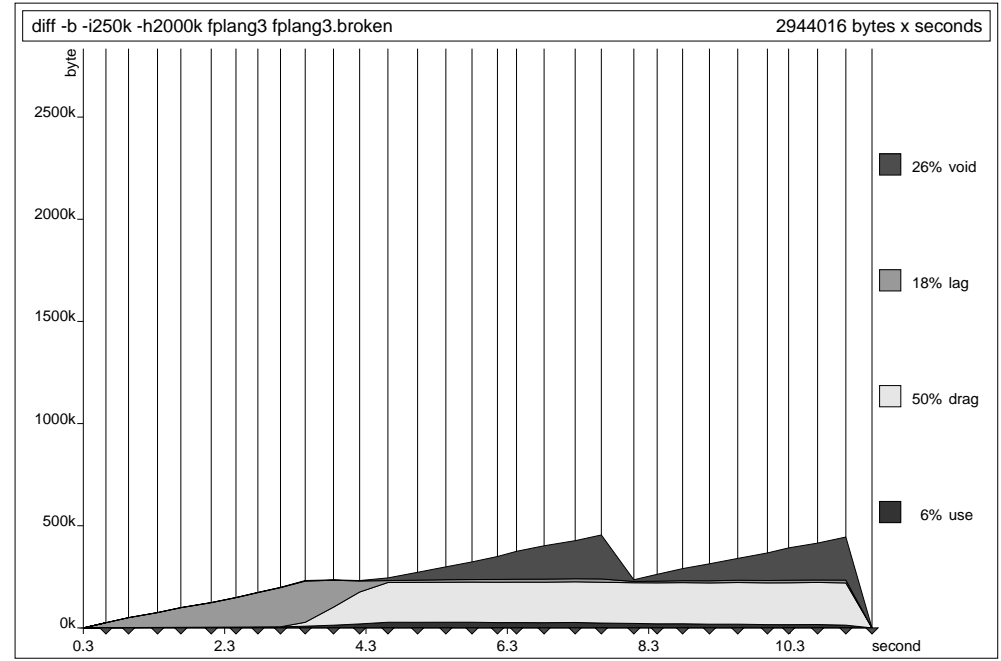

Fig. 21. Profile of a lazier diff (cf. Figure 20).

only memory allocation: for most applications the allocation count is just another run-time clock; it is no guide to the continuing size and content of the live heap.

The earliest 'true' memory-profiling tools were not for use by programmers wishing to improve their programs. Rather they were developed by researchers who wished to understand better the memory characteristics of implementation methods, with a view to improving them. For example, there was a published study of this kind in the late '70s for a SNOBOL4 system[4], and another a decade later for a fixed-combinator implementation of the lazy functional language SASL[3]. The emphasis in both cases was on summary statistics.

The first heap-profiler intended for functional programmers was developed in 1992; its design and use are described in [6]. The profiler recorded census data for static cell attributes only (constructor/closure or producer), and a separate program generated PostScript charts. Extended with whole-module producers and whole-type constructors, the profiler could be used to improve the spaceefficiency of large programs, such as compilers [7]. Limitations of a 'who produces what' view of the heap prompted the subsequent development of the nhc profiler, with its extensions to dynamic characteristics of heap cells: see [5] for details of retainer profiling ${ }^{4}$; see [9] for more about biographical profiling.

There has also been a concerted effort to develop profiling tools as part of the ghc optimising compiler project at Glasgow. Their profiler can attribute both time and space costs to 'cost centres' assigned either implicitly (eg. each

${ }^{4}$ Also lifetime profiling, now largely superceded by biographical profiling, but occasionally still a useful source of auxiliary information. 
function is a cost centre) or by explicit annotation of expressions - in which case the attributed costs are those expended in evaluating the entire expression (excluding free variables or separately annotated subexpressions). For profiling heap-memory cost-centres are treated as producers. See [11] or [10] for details of the ghc profiler.

Several other implementors have done work on their own heap-profiling tools, yet to be reported in the literature: for example, we know of such work by Appel (SML of New Jersey), Jones (Hugs/Gofer) and Tofte (ML with regionbased memory management).

\section{References}

1. Allison, L.: Lazy dynamic-programming can be eager Information Processing Letters 43 (1992) 207-212

2. Clack, C., Clayman, S., Parrott, D.: Lexical profiling: theory and practice. $J$. Functional Programming 5 (1995) 225-277

3. Hartel, P.H., Veen, A.H.: Statistics on graph reduction of SASL programs. Software - Practice and Experience 18 (1988) 239-253

4. Ripley, G.D., Griswold, R.E., Hanson, D.R.: Performance of storage management in an implementation of SNOBOL4. IEEE Transactions on Software Engineering SE-4 (1978) 130-137

5. Runciman, C., Röjemo, N.: New dimensions in heap profiling. J. Functional Programming 6 (1996) to appear

6. Runciman, C., Wakeling, D.: Heap profiling of lazy functional programs. J. Functional Programming 3 (1993) 217-245

7. Runciman, C., Wakeling, D.: Heap profiling of a lazy functional compiler. In Functional Programming, Glasgow 1992, Springer-Verlag Workshops in Computing (1993) 203-214

8. Röjemo, N.: Garbage collection, and memory efficiency, in lazy functional languages. Ph.D. thesis, Computer Sciences, Chalmers Univ. of Technology, Göteborg (1995)

9. Röjemo, N., Runciman, C.: Lag, drag, void and use - heap profiling and spaceefficient compilation revisited. In Proc. Intl. Conf. on Functional Programming (ICFP'96), ACM Press (1996) 34-41

10. Sansom, P.M.: Execution profiling for non-strict functional languages. Ph.D. thesis, Computing Science, Univ. of Glasgow (1994)

11. Sansom, P.M., Peyton Jones, S.L.: Time and space profiling for non-strict higherorder functional languages. Proc. ACM Conf. on Principles of Programming Languages (POPL'95), ACM Press (1995) 355-366 


\section{Appendix: summary of profiling options}

\section{Run-time flags}

Usage:

program profile-kind restrictions heap-size census-interval arguments

\begin{tabular}{|c|c|}
\hline \multicolumn{2}{|l|}{ profile-kind: } \\
\hline$-\mathrm{p}$ & producer by function \\
\hline$-\mathrm{m}$ & producer by module \\
\hline$-c$ & constructor \\
\hline$-r$ & retainer (can be given optional number for \\
\hline & maximum retainer-set size) \\
\hline$-b$ & biographic \\
\hline-1 & lifetime \\
\hline \multicolumn{2}{|c|}{ Zero or more restrictions can then be specified: } \\
\hline -pcomma separated list & restrict to functions in list \\
\hline -mcomma separated list & restrict to modules in list \\
\hline -ccomma separated list & restrict to constructors/closures in list \\
\hline -rcomma separated list & $\begin{array}{l}\text { restrict to nodes for which at least one retainer } \\
\text { is in the list }\end{array}$ \\
\hline -bcomma separated list & restrict to the phases in the list. \\
\hline$-1 \min -\max$ & restrict to nodes with lifetimes of at least $\mathrm{min}$, \\
\hline & and at most $\max$, censuses - one of the limits \\
\hline \multirow{2}{*}{\multicolumn{2}{|c|}{ Set heap size. The prefix $\mathrm{M}\left(10^{6}\right)$ or $\mathrm{k}\left(10^{3}\right)$ can be used: }} \\
\hline & \\
\hline -hsizeb & heap size in bytes \\
\hline -hsizew & heap size in words \\
\hline \multicolumn{2}{|c|}{$\begin{array}{l}\text { Set census interval. The prefix } \mathrm{M}\left(10^{6}\right) \text { or } \mathrm{k}\left(10^{3}\right) \text { can be used for allocation } \\
\text { intervals, } \mathrm{m}\left(10^{-3}\right) \text { for timed intervals: }\end{array}$} \\
\hline -iintervals & census interval in seconds \\
\hline -iintervalb & census interval in allocated bytes \\
\hline -iinterval $\mathrm{w}$ & census interval in allocated words \\
\hline \multicolumn{2}{|c|}{ Arguments to the Haskell program: } \\
\hline- & $\begin{array}{l}\text { stop decoding of run-time flags - only needed } \\
\text { if first argument starts with - }\end{array}$ \\
\hline
\end{tabular}




\section{Hp2graph}

Usage: hp2graph options file.hp

\begin{tabular}{|l|l|}
\hline Options: & $\begin{array}{l}\text { ignore trace bands - all those bands that to- } \\
\text { gether contribute less than the given percent- } \\
\text { age of overall cost }\end{array}$ \\
-tpercentage & use same scale as in the given aux-file \\
- $\mathrm{x}$ & produce an exploded graph \\
$-\mathrm{c}$ & omit profiling comments \\
$-\mathrm{y}$ & omit census lines \\
$-\mathrm{m}$ & omit garbage-collection marks \\
\hline
\end{tabular}

This article was processed using the $\mathrm{L}_{\mathrm{E}}^{\mathrm{A}} \mathrm{T}_{\mathrm{E}} \mathrm{X}$ macro package with LLNCS style 